\title{
Role of PET-CT in Aiding Diagnosis of Various Neurological Conditions - A Case Series
}

\author{
Harleen Singh Pabla르, P.R. Gokulakrishnan², Arunan Murali ${ }^{3}$, P.M. Venkata Sai ${ }^{4}$ \\ 1,2,3,4 Department of Radiodiagnosis, Sri Ramachandra Institute of Higher Education and Research, \\ Chennai, Tamilnadu, India.
}

\section{ABSTRACT}

\section{BACKGROUND}

PET-CT is an imaging modality which electronically detects positron-emitting radiopharmaceuticals in the human body and reveals its exact anatomical location. ${ }^{1}$ PET CT measures the metabolic and functional activity of living tissue noninvasively. ${ }^{1}$ This technology is utilized in diagnosis, planning treatment and predicting outcomes in various neurological conditions. ${ }^{1}$ Depending upon various patterns of FDG uptake in different parts of brain, 18FDG PET-CT allows us to differentiate between various types of dementia. ${ }^{2}$ PET CT allows tracking the course of disease and revealing the severity of the disease. ${ }^{2}$ In this article, we discuss the imaging findings of normal 18 FDG PET-CT of brain and 8 different neurological conditions with their corresponding brain PET-CT findings.

\section{METHODS}

To study the role of 18FDG-PET/CT in neurological conditions, we identified 8 different patients who underwent 18FDG-PET/CT imaging of brain for clinically suspected different neurological diseases at Department of Radiodiagnosis-Centre of Excellence (CERIS), SRIHER, Chennai, between 2015 and 2019. Siemens Biograph Horizon 16-slice PET/CT scanner with TrueV was used. Syngo.Via Version VB30A software was used. 18F- Fluorodeoxyglucose was the radiotracer used [Dose: 3-7 $\mathrm{mCi}$. After the scan, different patterns of 18 FDG uptake in the brain were analyzed in each of these patients.

\section{RESULTS}

18 FDG PET-CT showed reduced uptake in the epileptogenic foci in the brain. Alzheimer's disease showed decreased FDG uptake in bilateral precuneus, posterior cingulate region, parietal cortex and frontal cortex. Fronto-temporal dementia revealed reduced FDG uptake in anterior cingulate gyrus and anterior temporal lobe. Primary progressive aphasia showed asymmetrical reduced metabolic activity in the bilateral frontal and temporal lobes. Progressive supranuclear palsy revealed reduced metabolic activity in bilateral paramedian frontal region, head of caudate nuclei and midbrain; Multi systemic atrophy showed reduced metabolic activity in midbrain, pons, medulla oblongata and the cerebellum; AIDS related dementia showed global hypometabolism with preserved uptake in basal ganglia.

\section{CONCLUSIONS}

18FDG-PET/CT has a vital complementary role in the evaluation CNS disorders along with clinical examination, other imaging modalities like CT, MRI, and electroencephalogram (EEG). Radiologists should be aware of these different patterns of FDG uptake to aid the clinical diagnosis and early treatment.

\section{KEY WORDS}

18 FDG PET-CT, 18FDG Uptake, Hypometabolism, PET-CT Brain
Corresponding Author:

Dr. Harleen Singh Pabla,

R. No. 430, Boys Hostel, G-Block,

Sri Ramachandra Institute of Higher

Education and Research, Chennai,

Tamilnadu, India.

E-mail: harleenpabla@gmail.com

DOI: $10.14260 /$ jemds/2021/97

How to Cite This Article:

Pabla HS, Gokulakrishnan PR, Murali A, et al. Role of pet-CT in aiding diagnosis of various neurological conditions - a case series. J Evolution Med Dent Sci 2021;10(07):440-446, DOI: 10.14260/jemds/2021/97

Submission 26-07-2020,

Peer Review 10-12-2020,

Acceptance 17-12-2020,

Published 15-02-2021.

Copyright (C) 2021 Harleen Singh Pabla et al. This is an open access article distributed under Creative Commons Attribution License [Attribution 4.0 International (CC BY 4.0)] 


\section{BACKGROUND}

PET-CT is useful in quantification of cerebral metabolism and receptor binding. ${ }^{3}$ The only source of energy for the brain is the glucose. ${ }^{4}$ The brain utilizes approximately $20 \%$ of totalbody glucose metabolism in the fasting state. ${ }^{4}$ Hence normal brain shows intense uptake of 18FDG. ${ }^{4}$ The grey shows higher 18-FDG uptake than the white matter. ${ }^{4}$ Cerebral metabolic rate values for glucose are $40-60 \mathrm{micromol} / \mathrm{min} / 100 \mathrm{~g}$ for the grey matter and 15 micromol / $\mathrm{min} / 100 \mathrm{~g}$ for the white matter in the resting conditions. ${ }^{4}$ The normal grey to white matter activity ratio is 2.5 to $4.1{ }^{4}$ Data combined from PET CT and MRI can be used in localizing epileptogenic focus in patients with refractory epilepsy. ${ }^{1}$ Different software-based techniques are used to merge image data from different modalities i.e. registration and fusion techniques. ${ }^{5}$ Aligning two imaging data sets spatially with one another is known as registration. 5 Fusion is process of merging image data by overlaying two image data sets and then seeing them as one image. ${ }^{5}$ PET MRI fusion is performed by using rigid registration technique. ${ }^{6}$ This technique further involves utilization of two main strategies. ${ }^{6}$ First strategy involves recognition of identical structures in both image sets and then reduction of a "distance measure" between them. ${ }^{6}$ The matching criterion in second strategy involves voxel-per-voxel similarity measurement of complete 3 -dimensional data.$^{6}$ The "similarity measure" criterion directs this registration algorithm. ${ }^{6}$ Thus these image registration techniques form a single combined image in which the PET image data is shown in colour on top of gray scale CT / MR images. ${ }^{6}$ This fusion imaging provides exact anatomical information as well as reveals areas of increased or decreased metabolism. ${ }^{7}$

\section{METHODS}

To study the role of 18FDG-PET/CT in neurological conditions, we identified 8 different patients who underwent 18FDGPET/CT imaging of brain for clinically suspected different neurological diseases at Department of Radiodiagnosis-Centre of excellence (CERIS), SRIHER, Chennai, between 2015 and 2019. Equipment used: Imaging was performed on an integrated Siemens Biograph Horizon16-slice PET/CT scanner with TrueV with scanning of the Brain; CT scanning was performed without intravenous contrast material. Software used: Syngo.Via Version VB30A; Radiotracer used: 18FFluorodeoxyglucose [Dose: 3-7 $\mathrm{mCi}$ ]. PET-CT protocol included: Patient's clinical history obtained and routine screening done; Informed consent obtained; After overnight fasting [at least 4 to 6 hours], patients were changed into appropriate robes; Adequate oral hydration was advised; Blood glucose level was measured and recorded before injection of radiotracer; Appropriate radiotracer dose was taken, recorded and injected intravenously via peripheral cannula; Time of injection was also recorded; Patient was allowed to rest quietly for $45-60$ minutes in a shielded room for allowing FDG uptake into the tissues; Patients were made to rest in supine or recumbent position to reduce the FDG uptake in muscles. After the scan, different patterns of 18 FDG uptake in the brain were analyzed in each of these patients.

\section{RESULTS}

On 18FDG PET CT scan, various neurological diseases show different regions of reduced 18 FDG Uptake in the Brain as shown in table 1. Metabolic changes take place before the anatomical changes are seen on imaging; hence PET / CT has slight edge over other imaging modalities in conditions like memory, speech disturbances, epilepsy and movement disorders.

\section{DISCUSSION}

\section{Normal PET-CT of Brain}

In normal individuals, various brain regions poses different 18 FDG uptake. ${ }^{4}$ Higher 18FDG uptake is noted in frontal, parietal and occipital areas as compared to the temporal cortex. ${ }^{4}$ Slightly higher uptake is noted in basal ganglia as compared to the cortex. ${ }^{4}$ In normal individuals, focal regions of higher 18 FDG uptake are noted specifically in frontal eye fields, visual cortex and posterior cingulate cortex.. Relatively reduced 18FDG uptake is seen in medial temporal cortex, including the hippocamp areas as compared to the neocortex. ${ }^{4}$ Homogeneous and symmetrical 18-FDG uptake is seen in a normal brain. ${ }^{4}$ However, few areas of the brain such as Wernicke area, the frontal eye fields and the angular gyrus may show slight asymmetries in the uptake of 18-FDG. ${ }^{4}$

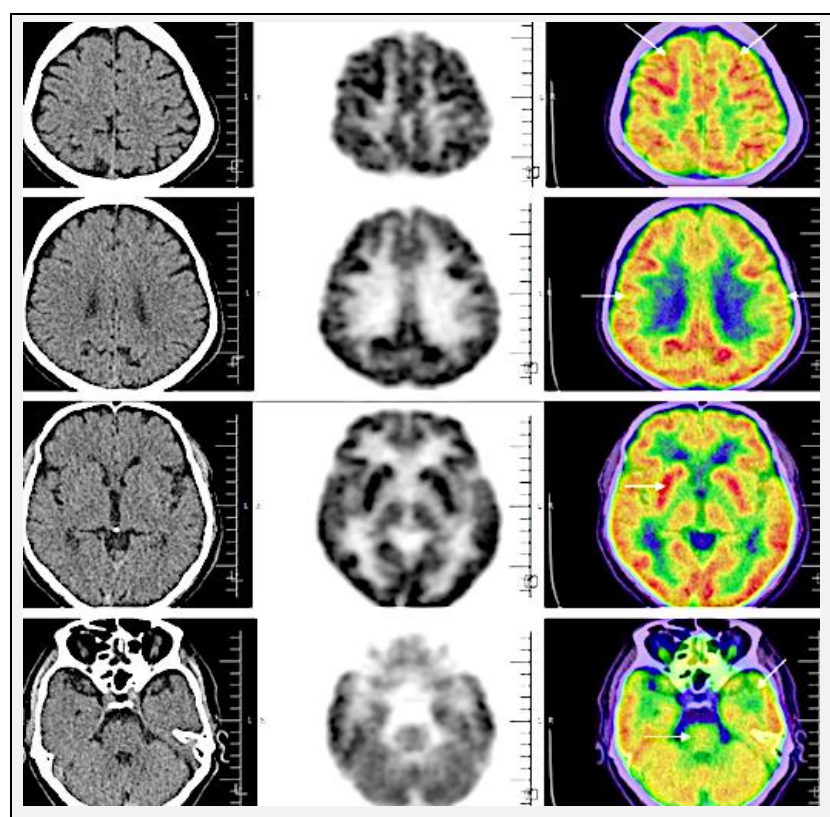

Figure 1. Selected Axial Sections of 18FDG PET CT of Brain Showing [White Arrows] Normal 18 FDG Uptake by the Brain; Red Regions Depict the Highest 18 FDG Uptake, Green Regions Depict Intermediate 18 FDG Uptake and Blue Regions Depict Least 18 FDG Uptake in the Brain

\section{Epilepsy}

In majority of the epilepsy cases, PET-CT is done during the interictal phase. ${ }^{8}$ Usually focal hypo metabolism is noted in interictal 18 FDG-PET images. ${ }^{8}$ It is difficult to perform ictal PET studies because of unpredictable nature of majority of seizures and their short time duration. ${ }^{8}$ Moreover there is a long uptake period of 18 FDG by the brain, approximately 30 - 
45 minutes after injection which may lead to complex pattern of increased and decreased metabolism. ${ }^{8}$ In postictal phase, PET-CT can reveal a complex pattern of increased and reduced metabolism. ${ }^{8}$ However, either increased or decreased metabolism can be seen during postictal phase, depending on the time of injection after seizure. ${ }^{8} 18$ FDG-PET is very useful for pre-surgical localization of epileptogenic foci, especially in patients with medically refractory partial seizures who have normal EEG and MRI study. ${ }^{8}$

\section{Case 1}

Clinical History

A 13-year-old male, a known case of seizure disorder visited Department of Radiodiagnosis-Centre of excellence (CERIS), SRIHER, Chennai with complaints of 4 to 5 episodes of focal seizures per month. The patient started having these seizure episodes at the age of 3 years. The seizure episodes predominantly occurred during night time. No history of fever or trauma. No history of loss of consciousness or giddiness. Antenatal history was uneventful. Developmental milestones were appropriate for age. The patient was on regular antiepileptics but discontinued since last 1 year. General physical examination was normal. All baseline laboratory investigations were normal. CT brain scan done in 2010 showed no significant abnormality. EEG done in 2015 showed abnormal epileptiform activity. MRI brain scan done in 2019 showed no significant abnormality. PET-CT brain was indicated for localization of epileptogenic foci.
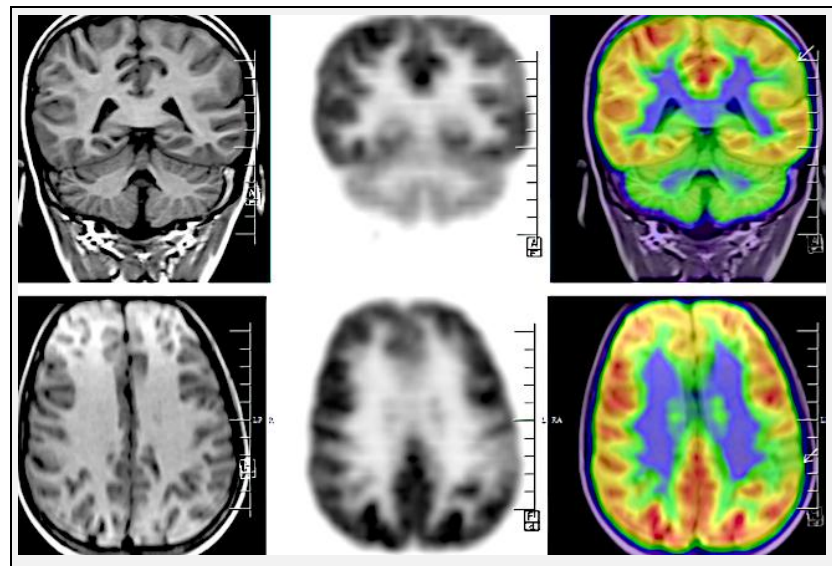

Figure 2. Selected Coronal and Axial Sections of 18-FDG PET / CT of Brain with MRI Fusion Showing [White Arrows] Focal Cortical Hypometabolism in the Left Frontal Lobe, in the Inferior Frontal Gyrus

\section{Case 2}

Clinical History

A 10-year-old, developmentally normal, female child visited Department of Radiodiagnosis-Centre of excellence (CERIS), SRIHER, Chennai with history of multiple transient ischemic attacks (TIA) since 5 years of age. The patient had $4-5$ episodes of (TIA) per day with loss of consciousness lasting for 2 minutes. The child was diagnosed to have Moyamoya disease and refractory seizures at 8 years of age and was started on anti-epileptics. For past 4 months there was history of reduced transient ischemic attacks. Recently there was 1 episode of transient ischemic attack, associated with seizures in the last 4 months. No history of fever or trauma. Birth history was uneventful. Developmental milestone were appropriate for age. General physical examination was normal. MRI brain scan showed focal gliosis in right frontal lobe and left posterior temporal lobe, which was suggestive of chronic infarct. MRI brain also showed high grade stenosis of bilateral intracranial internal carotid arteries with occlusion of bilateral middle and anterior cerebral arteries and multiple collaterals noted in thalamus and basal ganglia-features consistent with Moyamoya disease-stage III (Suzuki Staging). Indication of PET-CT brain was to localize the epileptogenic foci.

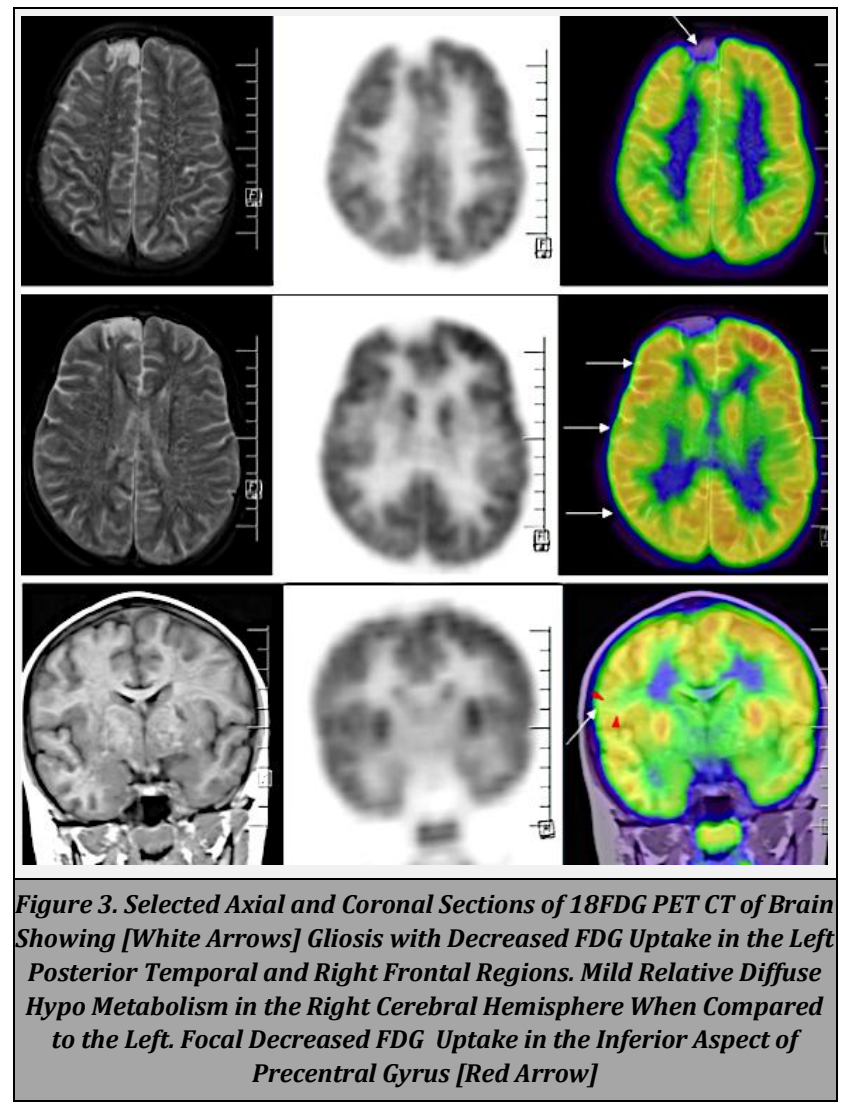

Dementia - Alzheimer's Disease (AD)

In Alzheimer's disease 18 FDG PET CT shows characteristic reduced uptake in temporoparietal region. ${ }^{3}$ With the progression of disease, reduced FDG uptake is noted in frontal cortices whereas normal metabolism is observed in the striatum, thalamus, primary sensorimotor cortices, visual cortices, and cerebellum. ${ }^{9}$ Severity of dementia is seen to correlate with degree of hypometabolism. ${ }^{3}$ The reason behind reduced glucose metabolism in $\mathrm{AD}$ is the combined effect of decreased synaptic activity and neuronal cell loss. ${ }^{3}$ FDG PET CT can detect AD even before the symptoms become evident. ${ }^{3}$ Reduced metabolism in the entorhinal cortex of elderly individuals with normal cognition can help in predicting the progression to $\mathrm{AD}$ and mild cognitive impairment. ${ }^{3}$

Case 3

Clinical History

A 60-year-old female visited Department of RadiodiagnosisCentre of excellence (CERIS), SRIHER, Chennai with 
complaints of memory loss since one and half years. No history of fever, trauma, loss of consciousness, giddiness or weight loss. No history of headache. Appetite was normal. Bowel and bladder habits were normal. No history of diabetes, hypertension or any other co-morbidities. No past surgical history was present. No history of any prior medications. General physical examination was normal. Neurological examination was normal. All baseline laboratory investigations showed no significant abnormality. In view of memory loss, MRI brain scan was done, which showed age related diffuse cerebral atrophy with bilateral frontal periventricular white matter small vessel ischemic changes. PET-CT brain was indicated for further evaluation.

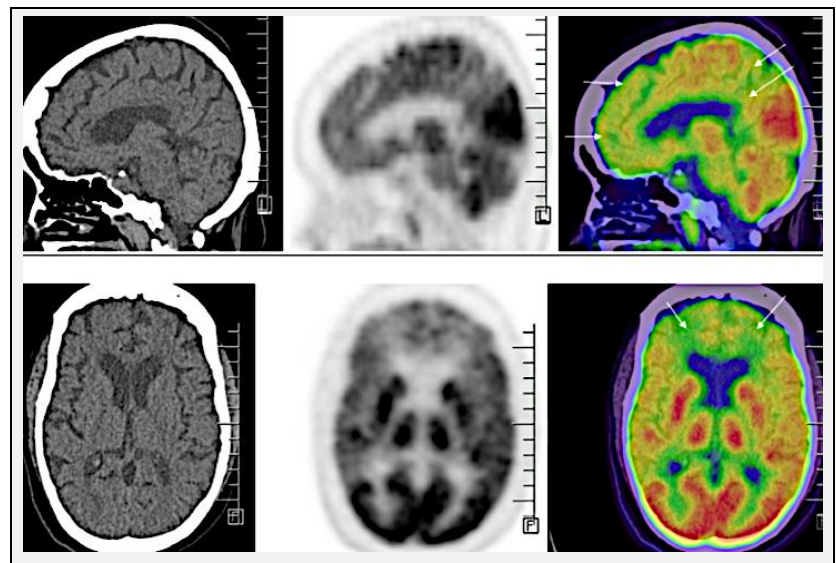

Figure 4. Selected Sagittal and Axial Sections of 18FDG PET CT of Brain Showing [White Arrows] Reduced FDG Uptake in Bilateral Precuneus, Posterior Cingulate Region, Parietal Cortex and Frontal CortexSuggestive of Alzheimer's Disease

\section{Frontotemporal Dementia}

In patients with frontotemporal dementia, 18-FDG PET / CT shows reduced FDG uptake in the frontal and temporal lobes. ${ }^{3}$ Dementia with lewy bodies (DLB) consists of changes similar to $\mathrm{AD}$ on FDG PET CT with additional reduced metabolism in the primary and associative visual cortices. ${ }^{3}$ Multi-infarct dementia reveals multiple focal areas of hypo metabolism on FDG PET CT. ${ }^{3}$ The extent of these focal areas of hypo metabolism seen in multi-infarct dementia is usually greater than the actual pathology seen on postmortem examination. ${ }^{3}$ This occurs probably due to degeneration of axons after the infarct with disconnection of remote structures. ${ }^{3}$

\section{Case 4}

Clinical History

A 64-year-old male, known case of type 2 diabetes mellitus, hypertension, dyslipidemia, post CABG (2009) status visited Department of Radiodiagnosis-Centre of excellence (CERIS), SRIHER, Chennai with chief complaints of memory loss for the past 4 months. History of increased day time sleepiness was present. Patient was a known smoker and non-alcoholic. No history of loss of consciousness, giddiness or palpitations. No history of trauma or fever. General physical examination was normal. Baseline laboratory parameters were normal except raised cholesterol. Indication for PET-CT brain for further evaluation of memory loss.

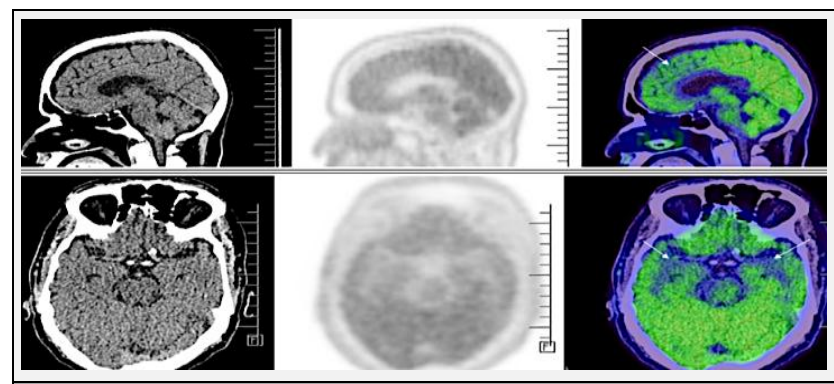

Figure 5. Selected Sagittal and Axial Sections of 18FDG PET CT of Brain Showing [White Arrows] Reduced FDG Uptake in the Anterior Cingulate Gyrus and Anterior Temporal Lobe -Suggestive of Frontotemporal Dementia

Primary Progressive Aphasia (PPA)

In patients with PPA, 18 FDG PET CT reveals progressive decline in metabolism in the left cerebral cortex including the temporal parts of language network i.e. Wernicke's area. ${ }^{10}$ Reduced metabolism is seen in inferior parietal cortex in the later stages of the disease. ${ }^{10}$ In the initial stages glucose hypo metabolism is noted in left anterior part of temporal region and then further involvement of left frontoinsular region and temporoparietal network is seen. ${ }^{10} \mathrm{PPA}$ is considered as a part of frontotemporal dementia group, however significant overlaps are seen in the later part of disease with the involvement of parietal region. ${ }^{10}$

\section{Case 5}

Clinical History

A 58-year-old male visited Department of RadiodiagnosisCentre of excellence (CERIS), SRIHER, Chennai with complaints of difficulty in talking and slurring of speech initially, which was followed by total inability to talk. No history of weakness and seizures. No history of diabetes and hypertension. General physical examination was normal. Local examination showed fasciculations involving tongue, left shoulder and left thigh. Oral examination revealed decreased palatal movements and reduced gag reflex. Jaw jerk was brisk. All baseline laboratory investigations were within normal limits. Pulmonary function tests were normal. Echo and ECG studies were normal. MRI-Cervical spine screening showed cervical and lumbar spondylosis. Indication of PET-CT for further evaluation of aphasia.

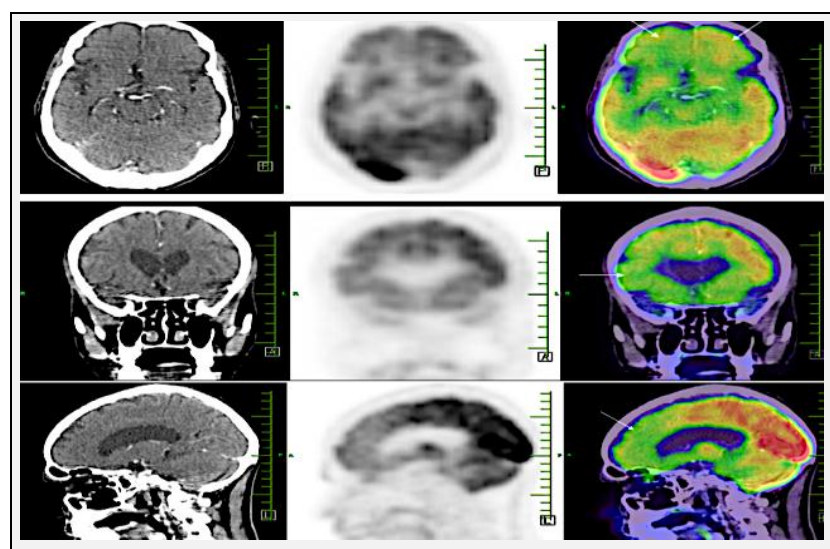

Figure 6. Selected Axial, Sagittal and Coronal Sections of 18FDG PET CT of Brain Showing [White Arrows] Asymmetrical Reduced Metabolic Activity seen Involving Bilateral Frontal (Right > Left) and Right Temporal Region Suggestive of Primary Progressive Aphasia 
Progressive Supranuclear Palsy

PSP is a form of neurodegenerative disease which characteristically involves basal ganglia and brain stem. ${ }^{11}$ Only advanced stages of PSP reveal abnormalities on MRI study. ${ }^{11}$ In patients with PSP, 18 FDG PET CT shows hypo metabolism in upper brainstem, caudate nuclei, insula cortices and frontal cortex.11 18 FDG PET CT can be used to differentiate Parkinson's disease and atypical Parkinsonism in the early stages. ${ }^{11}$

\section{Case 6}

\section{Clinical History}

A 52-year-old male patient visited Department of Radiodiagnosis-Centre of excellence (CERIS), SRIHER, Chennai with complaints of difficulty in walking and maintaining balance with history of frequent falls. Patient was treated for the same at a local hospital, however his condition did not improve. Patient also complained of tremors involving his right lower limb for which he was started on oral medications (details not known) but showed no improvement. Patient also developed difficulty in opening eyes for which he had taken BOTOX treatment multiple times, but his condition did not improve. Later he also complained of difficulty in speech, more in the form of dysarthria. Then the patient developed stiffness of all four limbs. Patient is a known case of type 2 diabetes mellitus on regular treatment. Neurological examination showed frontal lobe release signs were present. Patient showed eyelid apraxia with blepharospasm. Eye movements showed evidence of restricted vertical and horizontal gaze. Hypomimic facies noted. Sensory system was normal. No evidence of cerebellar signs. No evidence of raised intracranial tension. The patient had fast gait with reduced arm swing and axial rigidity. Baseline laboratory investigations were normal. MRI brain scan showed small vessel ischemic disease, mildly thinned out M3 segment of right middle cerebral artery and age-related cerebral atrophy. PET-CT brain was indicated for further neurological evaluation.

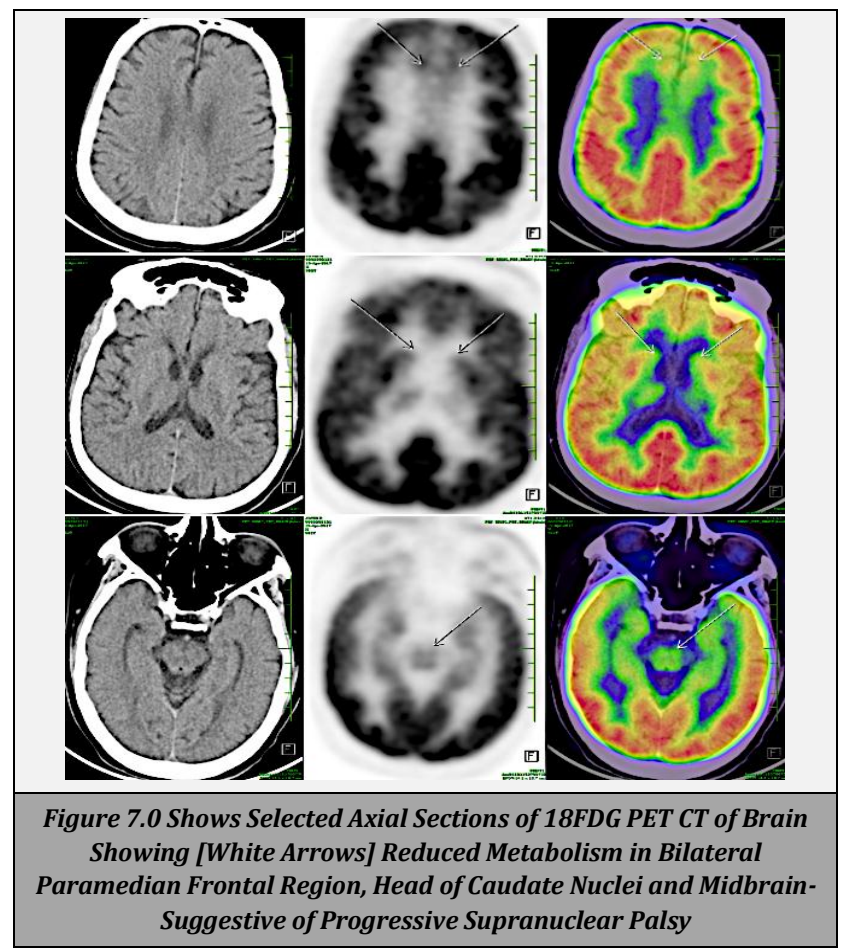

Multi Systemic Atrophy (MSA)

18-FDG PET / CT shows patterns of regional reduced metabolism that corresponds to areas of atrophy and T2 signal alteration in MRI studies. ${ }^{12}$ Patients with MSA-P reveal relatively symmetric hypo metabolism in putamen on 18FDG PET CT, whereas patients with MSA-C show glucose hypo metabolism in cerebellar hemispheres and middle cerebellar peduncles. ${ }^{12}$ Patients without any clinical signs of cerebellar dysfunction may also show cerebellar hypometabolism. ${ }^{12}$ Sometimes patients with MSA-P may also show reduced FDG uptake in cerebellar hemispheres.12 18FDG PET CT can be used to differentiate MSA from Parkinson disease as the latter shows normal putaminal and cerebellar metabolism. ${ }^{12}$

\section{Case 7}

\section{Clinical History}

A 54-year-old male patient visited Department of Radiodiagnosis-Centre of excellence (CERIS), SRIHER, Chennai with complaints of giddiness on getting up from supine position along with unsteadiness of gait since 2 years of duration. History of slurred speech was present since 3 months of duration with progressive deterioration. History of erectile dysfunction and increased urinary frequency with complaints of constipation was present since 6 months. No history of trauma, head injury, fever, neck stiffness or vomiting. No history of loss of consciousness or seizures. No history of weakness of limbs. The patient is a known case of type 2 diabetes mellitus, hypothyroidism and on regular treatment. General physical examination was normal. Neurological examination showed finger to nose and finger to finger test incoordination was present. Heel-to- Shin test was positive. Dysdiadochokinesia was present bilaterally. Gait ataxia and bradykinesia was present. Romberg's test was positive. Baseline lab investigations were normal except elevated blood glucose levels.

MRI brain showed atrophy of medullary olive, pons and pontine belly with hot cross bun appearance noted. Minimal thinning of corpus callosum with dilatation and splaying of lateral ventricles noticed. MR angiography showed aplasia of P4 segment of right posterior cerebral artery and hypo plastic A1 segment of right anterior cerebral artery-features of multi system atrophy-olivoponto-cerebellar type. PET-CT brain was indicated for further evaluation.

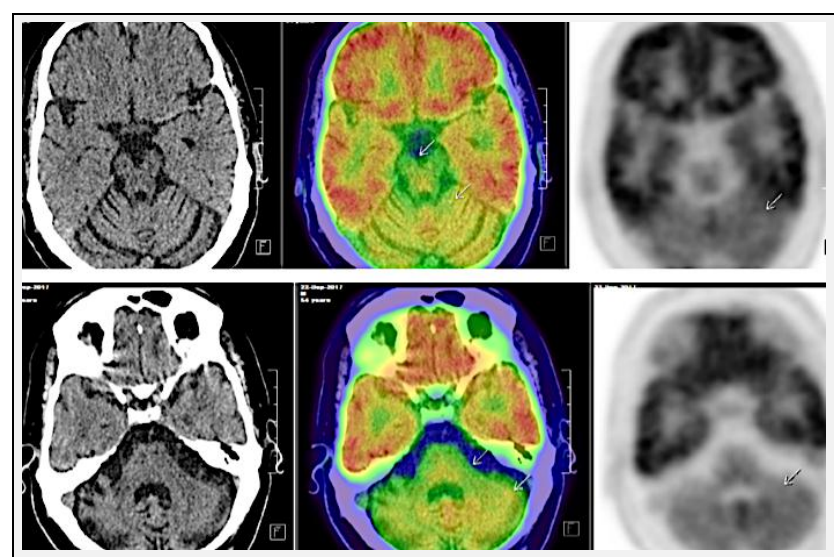

Figure 8. Selected Axial Sections of 18FDG PET CT of Brain Showing [White Arrows] Hypo Metabolic Changes in the Mid Brain, Pons, Medulla Oblongata and the Cerebellum - Suggestive of Multi Systemic Atrophy \{Cerebellar Type\} 


\section{AIDS Related Dementia}

In patients with AIDS related dementia, 18FDG PET CT reveals globally reduced cortical metabolism mainly in bilateral frontal, parietal and temporal cortex. ${ }^{13}$ However, normal FDG uptake is noted in the basal ganglia. ${ }^{13}$

\section{Case 8}

\section{Clinical History}

A 38-year-old female, known case of PLHA (People living with AIDS), on regular medications, visited Department of Radiodiagnosis-Centre of excellence (CERIS), SRIHER, Chennai with complaints of fever and loose stools since 1 month. History of nausea, loss of weight and loss of appetite were present. No history of abdominal pain or melena. Patient is a known case of diabetes mellitus for the past 3 months on regular medications and known case of hypertension for the past 2 years. No history of tuberculosis, asthma, or epilepsy. General physical examination showed hepatomegaly. PCR for HIV I (quantitative) was 2697 IU. CD4 counts were 55. HBsAg and HCV were negative. Peripheral smear examination showed macrocytic anemia. After obtaining all the baseline investigations a working diagnosis of PLHA with disseminated tuberculosis / malignancy was made. To rule out infiltrative disorder, ultrasound guided liver biopsy was done, and it showed steatohepatitis. In view of lower limb proximal muscle weakness, MRI whole spine was done, which showed disc bulge at level of L4 - L5, L5 - S1 vertebral levels. PET-CT brain was indicated for further evaluation.

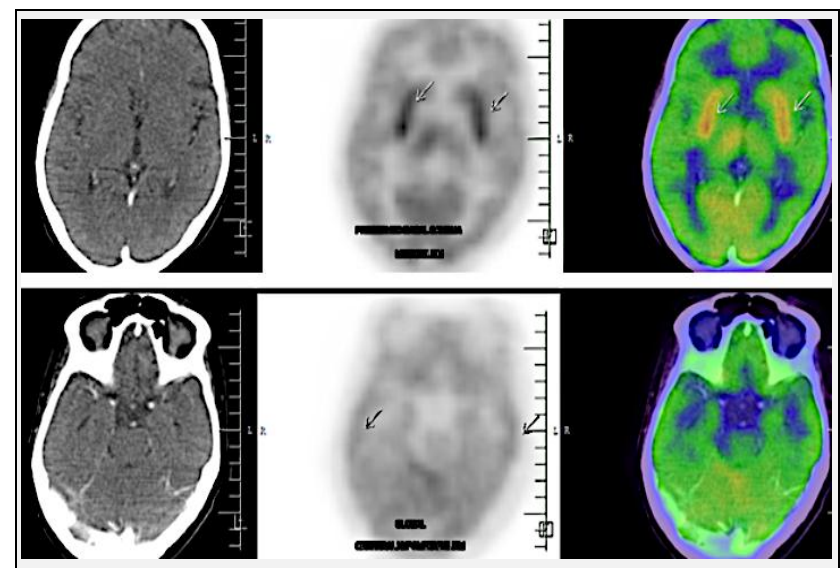

Figure 9. Selected Axial Sections of 18FDG PET CT of Brain Showing [White Arrows] Global Hypo Metabolism with Preserved Uptake in Basal Ganglia - Suggestive of AIDS Related Dementia

\begin{tabular}{|c|c|}
\hline $\begin{array}{l}\text { Neurological } \\
\text { Condition }\end{array}$ & Area of Reduced 18 FDG Uptake in the Brain \\
\hline Epilepsy & Epileptogenic foci \\
\hline Alzheimer's Disease & $\begin{array}{l}\text { Bilateral precuneus, posterior cingulate region, parietal cortex } \\
\text { and frontal cortex }\end{array}$ \\
\hline $\begin{array}{l}\text { Fronto-Temporal } \\
\text { Dementia }\end{array}$ & Anterior cingulate gyrus and anterior temporal lobe \\
\hline $\begin{array}{l}\text { Primary Progressive } \\
\text { Aphasia }\end{array}$ & $\begin{array}{l}\text { Asymmetrical reduced metabolic activity seen involving } \\
\text { bilateral frontal and temporal lobes }\end{array}$ \\
\hline $\begin{array}{c}\text { Progressive } \\
\text { Supranuclear Palsy }\end{array}$ & $\begin{array}{l}\text { Bilateral paramedian frontal region, head of caudate nuclei } \\
\text { and midbrain }\end{array}$ \\
\hline $\begin{array}{l}\text { Multi Systemic } \\
\text { Atrophy }\end{array}$ & Midbrain, pons, medulla oblongata and the cerebellum \\
\hline Aids Related Dementia & $\begin{array}{l}\text { Global hypometabolism with preserved uptake in basal } \\
\text { ganglia }\end{array}$ \\
\hline \multicolumn{2}{|r|}{ Table 1} \\
\hline
\end{tabular}

\section{CONCLUSIONS}

18FDG PET CT scan has a vital complementary role in evaluation CNS disorders along with clinical examination, other imaging modalities like CT, MRI and electroencephalogram (EEG). Radiologists should be aware of these different patterns of FDG uptake to aid the clinical diagnosis and early treatment.

\section{Abbreviations}

FDG = 2 - [fluorine - 18] Fluoro-2-Deoxy-d-Glucose, PSP = Progressive Supranuclear Palsy, MSA = Multiple System Atrophy, MSA-C = Cerebellar Ataxia Type MSA, MSA-P = Parkinsonian Type MSA, AD = Alzheimer's disease, $\mathrm{DLB}=$ Dementia with Lewy bodies.

Data sharing statement provided by the authors is available with the full text of this article at jemds.com.

Financial or other competing interests: None.

Disclosure forms provided by the authors are available with the full text of this article at jemds.com.

\section{REFERENCES}

[1] Kumar S, Rajshekher G, Prabhakar S. Positron emission tomography in neurological diseases. Neurol India 2005;53(2):149-55.

[2] Tripathi RP. Recent trends in molecular imaging : PET/CT in neurology. Ann Natl Acad Med Sci (India) 2014;50(1\&2):34-44.

[3] Tai YF, Piccini P. Applications of positron emission tomography (PET) in neurology. J Neurol Neurosurg Psychiatry 2004;75(5):669-76.

[4] Berti V, Mosconi L, Pupi A. Brain: normal variations and benign findings in fluorodeoxyglucose- PET/computed tomography imaging. PET Clin 2014;9(2):129-40.

[5] Giesel FL, Mehndiratta A, Locklin J, et al. Image fusion using CT, MRI and PET for treatment planning, navigation and follow up in percutaneous RFA. Exp Oncol 2009;31(2):106-14.

[6] Zaidi H, Montandon ML, Alavi A. The clinical role of fusion imaging using PET, CT and MR imaging. Magn Reson Imaging Clin N Am 2010;18(1):133-49.

[7] Kampe KKW, Rotermund R, Tienken M, et al. Diagnostic value of positron emission tomography combined with computed tomography for evaluating critically ill neurological patients. Frontiers in Neurology 2017;8:33.

[8] Sarikaya I. PET studies in epilepsy. Am J Nucl Med Mol Imaging 2015;5(5):416-30.

[9] Marcus C, Mena E, Subramaniam RM. Brain PET in the diagnosis of Alzheimer's disease. Clin Nucl Med 2014;39(10):e413-22.

[10] Koç ZP, Kara PPÖ, Doğu O. F-18 FDG PET/CT image of alzheimer like primary progressive aphasia. Glob Imaging Insights 2017;2(4):1-2.

[11] Belezia AB, Marussi VHR, Yared J, et al. PET-CT imaging in a patient with progressive supranuclear palsy. Arq Neuropsiquiatr 2015;73(4):364-5. 


\section{Jemds.com}

[12] Broski SM, Hunt CH, Johnson GB, et al. Structural and functional imaging in parkinsonian syndromes. Radiographics 2014;34(5):1273-92.

[13] Verma P, Asopa RV. Incidental global hypometabolism in the brain of patient with AIDS- related dementia seen on
Original Research Article

18F- fluorodeoxyglucose positron emission tomography/computed tomography. Indian J Nucl Med 2018;33(1):73-5. 